\title{
Production of Dihydroisocoumarins in Fusarium verticillioides by Swapping the Ketosynthase Domain of a Fungal Iterative Polyketide Synthase Fum1p with That of Lovastatin Diketide Synthase
}

Materials. Fusarium verticillioides wild-type strain A0149 (FGSC number 7600) was used in the study. Plasmid vector pUCH2-8 that contains hygromycin B resistant gene for transformation selection was used to transform the fungus. V8 medium was used for conidia production, YPD medium for mycelia growth, and GYAM medium for metabolites production. ${ }^{1}$ Escherichia coli DH5- $\alpha$ was used as host for general DNA preparation, and plasmid preparation and DNA extraction were carried out with Qiagen kits (Valencia, CA).

Construction of Plasmid Vector pZXC-1w92-LDKS. The KS domain of LDKS was amplified by PCR from pWHM1602 ${ }^{2}$ with primer LDKS-c-FOR, $5^{\prime}$-ATA TAT CGC ATG CTG GAG GAA GGA GGG AGC-3' (underlined SphI site), and primer LDKS-c-REV, 5'-CGC AGC TGG TAC CTG GAT ATG AGT GTC CTT-3' (underlined KpnI site). The 1079 bp PCR product was cloned into $\mathrm{pUCH}-\mathrm{fKS}{ }^{3}$ at the SphI and KpnI sites to produce pZXC-1w92-LDKS (Figure S1). The fidelity of the replaced LDKS-KS domain and the fusion regions between FUM1 and LDKS-KS was confirmed by DNA sequencing. Thus, a region of 362 amino acid residues (from number 56 to 417) of Fum1p (AAD43562) was replaced by a region of 360 residues (from number 36 to 395) of LDKS (AAD34559). In this construct, the LDKS-KS is flanked by the upstream region (461 bp) and the downstream region (2645 bp) of the KS domain of FUM1.

Generation of $\boldsymbol{F}$. verticillioides Mutants. The protocol used to produce and transform protoplasts was described in our previous work. ${ }^{4}$ The transformation was mediated with PEG-8000 buffer (30\% PEG-8000; $10 \mathrm{mM}$ Tris-HCl, $\mathrm{pH}=8.0 ; 50 \mathrm{mM} \mathrm{CaCl}_{2}$ ) at room temperature for $30 \mathrm{~min}$, and transformants were selected on YPD plates containing with $150 \mu \mathrm{g} / \mathrm{mL}$ hygromycin B (Calbiochem, La Jolla, CA). The two-stage screening approach was used for identifying the putative mutants, which resulted first from a single crossover event and then from a double crossover event at the two homologous regions. In the first stage, we obtained 30 hygromycin resistant colonies, which grew on 11 YPD plates, from $1 \times 10^{8}$ protoplasts transformed with $3 \mu \mathrm{g}$ pZXC-1w92-LDKS (Figure S1). Among the colonies, six were analyzed by PCR and Southern blot. One (LD-41) was confirmed as a true single crossover mutant resulted from homologous recombination at the downstream region, while the other five were either resulted from a random insertion or ectopic recombination. In the second stage, 1400 single colonies derived from strain LD-41 were screened for the loss of hygromycin resistance, using the duplicating method. ${ }^{4}$ We obtained 41 colonies that could not grow in hygromycin-containing YPD, and the colonies were subjected to PCR analysis (Figure S1). Two strains (LD-12 and LD-22) were identified from the 
colonies to result from double crossover, and the rest were revertants (wild-type). The identity of strains was further confirmed by Southern blot analysis (Figure S1).

Isolation of Fungal Metabolites. Single conidia of the wild type strain as well the mutants were inoculated on GYAM plates ( $20 \mathrm{~mL}$ size per plate) and were allowed to grow in the dark condition for 3-4 weeks. The medium with the fungi were smashed and extracted with the equal volume of methanol for overnight. The crude extracts were analyzed by HPLC (Prostar, Model 210, Varian, Walnut Creek, CA) coupled to an evaporative laser scattering detector (ELSD2000, Alltech, Deerfield, IL), to determine the production of fumonisins or new metabolites prior to the preparative HPLC (Figure S2). The column used was Alltima C18LL (5 $\mu, 250$ x 4.6 mm i.d., Alltech, Deerfield, IL) and the HPLC conditions were the same as described previously. ${ }^{5}$ To isolate the new metabolites, the methanol extracts $(200 \mathrm{~mL})$ were diluted 5 times with water and then loaded onto a C-18 cartridge (Sep-Pak Vac $35 \mathrm{cc}-10 \mathrm{~g}$ ). The metabolites were eluted from the cartridge by $80 \%-100 \% \mathrm{MeOH}$ and purified by a preparative HPLC column (Alltima C18LL , 5 $\mu, 250 \times 10 \mathrm{~mm}$ i.d., Alltech) using the same solvent systems to yield compounds 1, 2, 3, and 4, which corresponds to peaks 1-4, respectively, in the analytic HPLC shown in Figure S2. Quantity accumulation of compounds 1-4 were achieved by repeating the above procedure. Fumonisin $\mathrm{B}_{1}$ and $\mathrm{B}_{2}$ were the major metabolites isolated from the wild type. The yield for $\mathrm{FB}_{1}$ and $\mathrm{FB}_{2}$ was 161.6 and $61.4 \mu \mathrm{g} / \mathrm{g}$ solid GYAM medium, respectively, after 22-day growth. In the KS-replaced mutants, the yield for compounds 1-4 was 129.9, 87.6, 12.3, and $11.9 \mu \mathrm{g} / \mathrm{g}$ solid GYAM medium, respectively, after 16-day growth.

Structural Determination of Fungal Metabolites. Optical rotations were measured on Autopol@ IV automatic polarimeter. NMR spectra were recorded on Bruker DRX Avance 400 or 500 instruments. High resolution TOFMS (negative ion mode) were measured on an Agilent Series 1100 SL equipped with an ESI source.

7-(E)-But-2-enyl-3,4-dihydro-6,8-dihydroxy-3R-(E)-pent-3-enylisochro-men-1one-6-sulphate (1): off-white powder, $[\alpha]_{\mathrm{D}}^{25}-13(\mathrm{MeOH}, \mathrm{c} 0.15)$; NMR, see Table S1; HRTOFMS $m / z 381.1025$ calcd for $\left[\mathrm{M}\left(\mathrm{C}_{18} \mathrm{H}_{21} \mathrm{O}_{7} \mathrm{~S}\right]\right.$ ], 381.1014\}.

7-Butyl-3,4-dihydro-6,8-dihydroxy-3R-(E)-pent-3-enylisochromen-1-one-6-sul phate (2): off-white powder, $[\alpha]_{\mathrm{D}}^{25}-11(\mathrm{MeOH}, \mathrm{c} 0.10)$; NMR, see Table S1; HRTOFMS m/z 383.1177 calcd for $\left[\mathrm{M}\left(\mathrm{C}_{18} \mathrm{H}_{23} \mathrm{O}_{7} \mathrm{~S}\right]\right.$ ], 383.1170 \}.

7-(E)-But-2-enyl-3,4-dihydro-6,8-dihydroxy-3R-(E)-pent-3-enylisochro-men-1one (3): off-white powder, $[\alpha]_{\mathrm{D}}^{25}-22(\mathrm{MeOH}$, c 0.08$)$ \{ref. 6 in SI: $[\alpha]_{\mathrm{D}}^{25}-28.0(\mathrm{MeOH}$, c 0.10$)$ \}; NMR, see Table S1; HRTOFMS m/z 301.1414 \{calcd for $\left[\mathrm{M}\left(\mathrm{C}_{18} \mathrm{H}_{21} \mathrm{O}_{4}\right]^{-}\right.$, $301.1445\}$.

7-Butyl-3,4-dihydro-6,8-dihydroxy-3R-(E)-pent-3-enylisochromen-1-one off-white powder, $[\alpha]_{\mathrm{D}}^{25}-25(\mathrm{MeOH}, \mathrm{c} 0.07)\left\{\right.$ ref. 6 in SI: $\left.[\alpha]_{\mathrm{D}}^{25}-28.3(\mathrm{MeOH}, \mathrm{c} 0.25)\right\}$; 
Supporting Information: Xiangcheng Zhu, Fengan Yu, Xing-Cong Li, and Liangcheng Du

NMR, see Table S1; HRTOFMS m/z 303.1599 \{calcd for $\left[\mathrm{M}\left(\mathrm{C}_{18} \mathrm{H}_{23} \mathrm{O}_{4}\right]-303.1602\right\}$. 
Supporting Information: Xiangcheng Zhu, Fengan Yu, Xing-Cong Li, and Liangcheng Du

Table S1. NMR Data of Compounds 1-4 in $\mathrm{CD}_{3} \mathrm{OD}(\delta, \mathrm{ppm})^{a}$

\begin{tabular}{|c|c|c|c|c|c|c|c|c|}
\hline \multirow[b]{2}{*}{$\mathrm{C} / \mathrm{H}$} & \multicolumn{2}{|c|}{1} & \multicolumn{2}{|c|}{2} & \multicolumn{2}{|c|}{3} & \multicolumn{2}{|c|}{4} \\
\hline & ${ }^{1} \mathrm{H}$ & ${ }^{13} \mathrm{C}$ & ${ }^{1} \mathrm{H}$ & ${ }^{13} \mathrm{C}$ & ${ }^{1} \mathrm{H}$ & ${ }^{13} \mathrm{C}$ & ${ }^{1} \mathrm{H}$ & ${ }^{13} \mathrm{C}$ \\
\hline 1 & - & 170.37 & - & 170.37 & - & 170.75 & - & 170.75 \\
\hline 3 & 4.55 & 79.17 & 4.55 & 79.17 & 4.51 & 78.75 & 4.51 & 78.79 \\
\hline 4 & 2.94 & 32.30 & 2.94 & 32.30 & 2.84 & 32.36 & 2.86 & 32.32 \\
\hline $4 a$ & - & 138.17 & - & 138.17 & - & 138.84 & - & 138.84 \\
\hline 5 & 7.05 & 110.28 & 7.05 & 110.28 & 6.23 & 106.07 & 6.23 & 105.68 \\
\hline 6 & - & 156.38 & - & 156.38 & - & 163.30 & - & 163.30 \\
\hline 7 & - & 118.94 & - & 118.94 & - & 112.89 & - & 112.89 \\
\hline 8 & - & 161.05 & - & 161.05 & - & 161.65 & - & 161.65 \\
\hline $8 \mathrm{a}$ & - & 103.99 & - & 103.99 & - & 99.38 & - & 99.38 \\
\hline 9 & $1.78 /$ & 34.26 & $1.78 /$ & 34.26 & $1.78 /$ & 34.32 & $1.78 /$ & 34.31 \\
\hline & 1.86 & & 1.86 & & 1.87 & & 1.90 & \\
\hline 10 & 2.21 & 27.50 & 2.21 & 27.50 & 2.22 & 27.53 & 2.22 & 27.53 \\
\hline 11 & 5.52 & 129.66 & 5.52 & 129.66 & 5.50 & 129.72 & 5.50 & 129.70 \\
\hline 12 & 5.52 & 125.63 & 5.52 & 125.63 & 5.50 & 125.56 & 5.50 & 125.58 \\
\hline 13 & 1.64 & 16.66 & 1.64 & 16.66 & 1.67 & 16.63 & 1.67 & 16.63 \\
\hline 14 & 3.34 & 25.78 & 2.70 & 22.41 & 3.25 & 24.95 & 2.61 & 21.72 \\
\hline 15 & 5.48 & 127.80 & 1.52 & 30.81 & 5.46 & 128.33 & 1.50 & 30.65 \\
\hline 16 & 5.48 & 125.11 & 1.38 & 22.54 & 5.46 & 124.20 & 1.36 & 22.37 \\
\hline 17 & 1.58 & 16.59 & 0.93 & 12.98 & 1.62 & 16.52 & 0.94 & 12.99 \\
\hline
\end{tabular}


A

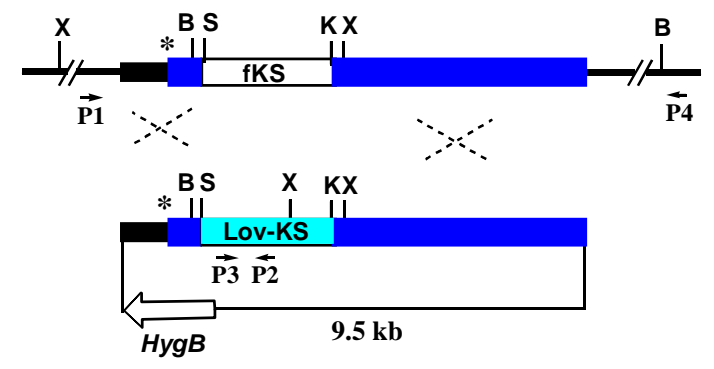

F. verticillioides

chromosome (FUM1)

pZXC-1w92-LDKS
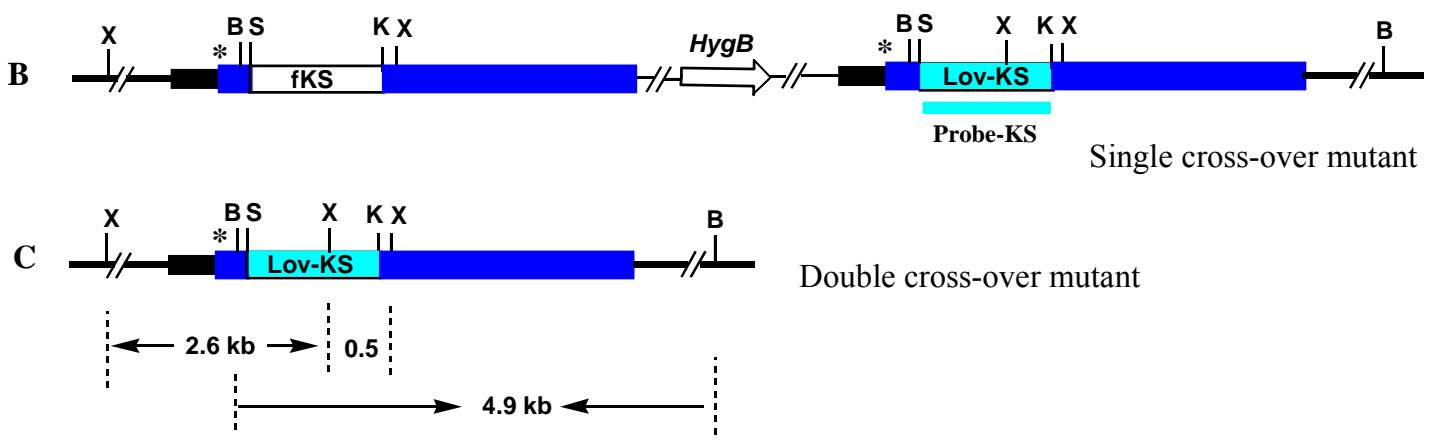

Double cross-over mutant

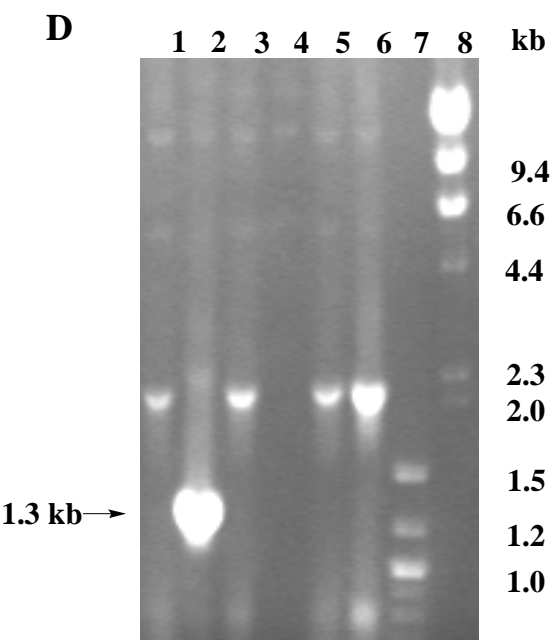

PCR Screen

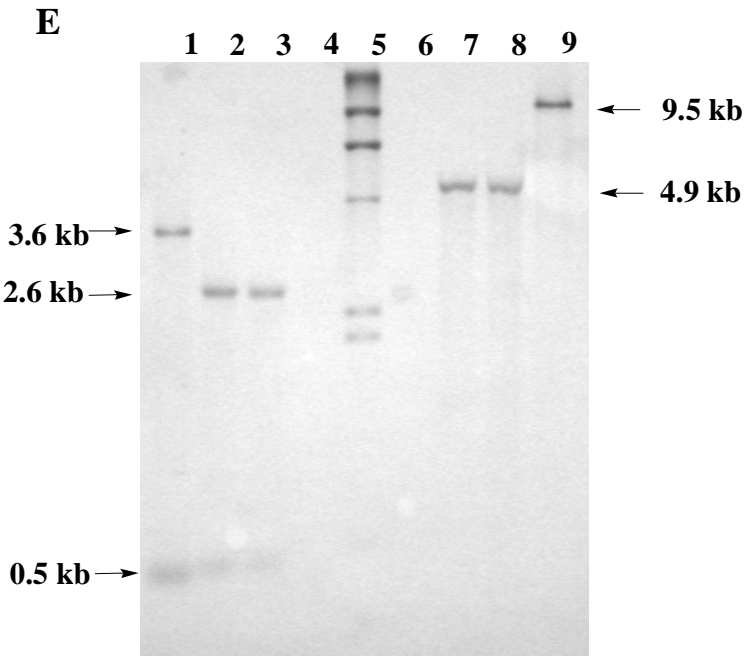

Southern Blot

Figure S1. The Screening and Confirmation of KS-replaced Mutants. (A) Homologous recombination between FUM1 on chromosome of F. verticillioides and the sequences on plasmid pZXC-1w92-LDKS. The "*" indicates the position of the start codon of FUM1. The positions for PCR primer binding sites in the screening of the mutants are indicated by small arrows. Abbreviations: B: BamHI; K: KpnI; S: SphI; $\mathrm{X}$ : XhoI; HygB, hygromycin B resistant gene. (B) Gene disruption mutants resulted from a single crossover at the downstream homologous region. (C) KS domain replaced mutant resulted from a second crossover at the upper homologous region. (D) Confirmation of the mutants by PCR (only showing part of the results using primers $\mathrm{P} 1 / \mathrm{P} 2)$. Lane-1 to 5, results from individual colonies that lost the hygromycin-resistance phenotype in the second selection, with one positive colony 
(LD-12) having the expect $1.3 \mathrm{~kb}$ PCR product; lane-6, from the wild type; lane-7 and 8, size markers. (E) Confirmation of mutants by Southern hybridization. The genomic DNA was digested with XhoI (lane- 1 to 4) or BamHI (lane-6 to 9) and probed with a DIG-labeled Lov-KS domain as shown in (C). The sizes of hybridizing signals are indicated with arrows. Lane-1 and 9, plasmid; lane-5, size markers; lane-2 and 8, mutant LD-22; lane-3 and 7, mutant LD-12; and lane-4 and 6, the wild-type. 


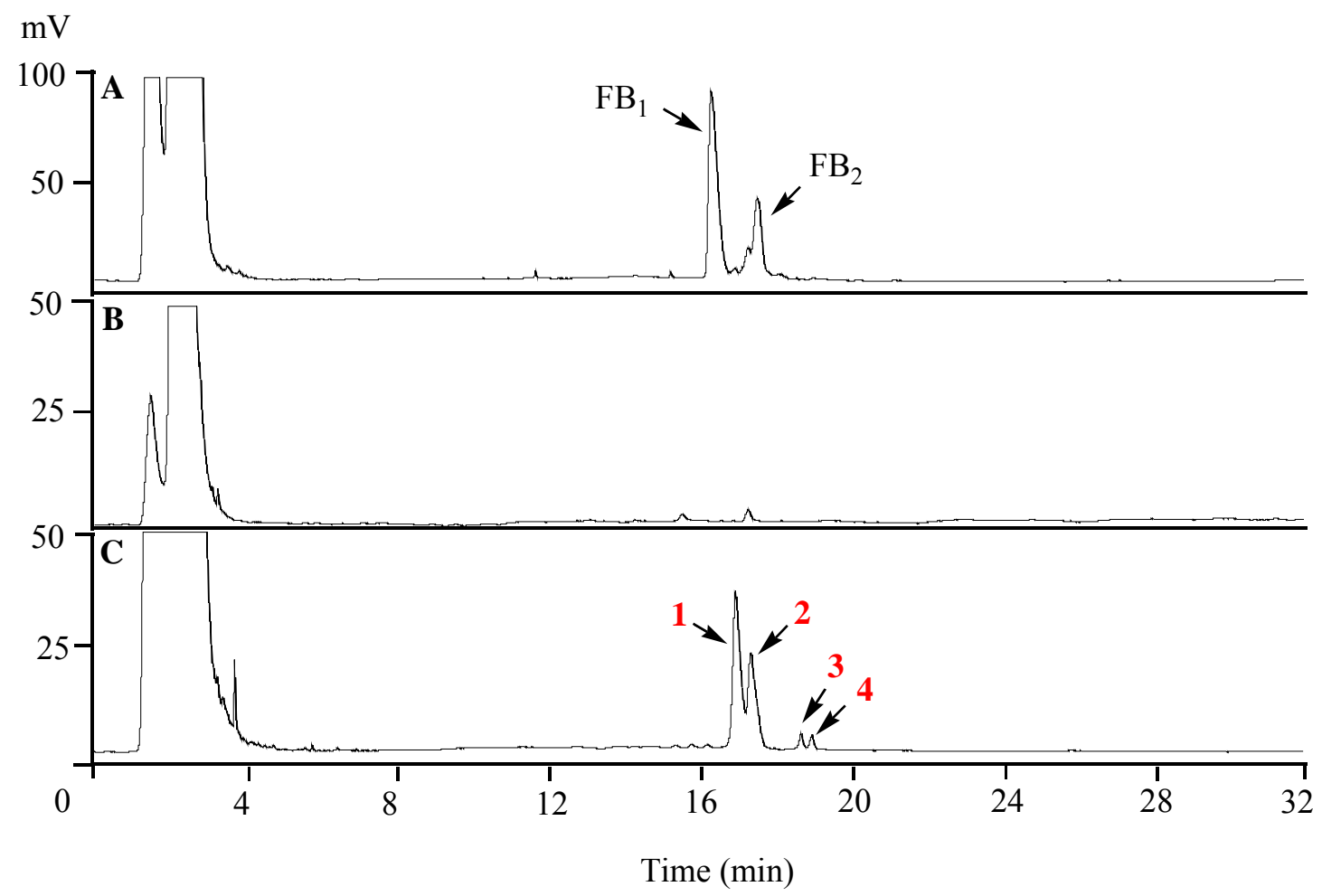

Figure S2. HPLC Analysis of Metabolites Produced by the Wild Type (A), the FUM1-disrupted Mutant Strain LD-41 (B), and the KS-replaced Mutant Strain LD-22 (C). Fumonisin $\mathrm{B}_{1}\left(\mathrm{FB}_{1}\right)$ and $\mathrm{B}_{2}\left(\mathrm{FB}_{2}\right)$ are the major metabolites produced by the wildtype. 
Supporting Information: Xiangcheng Zhu, Fengan Yu, Xing-Cong Li, and Liangcheng Du

\section{References:}

(1) Proctor, R. H.; Desjardins, A. E.; Plattner, R. D.; Hohn, T. M. Fungal Genet Biol 1999, 27, $100-112$

(2) Kennedy, J.; Auclair, K.; Kendrew, S. G.; Park, C.; Vederas, J. C.; Hutchinson, C. R. Science 1999, 284, 1368-1372.

(3) Zhu, X.; Yu, F.; Bojja, R. S.; Zaleta-Rivera, K.; Du, L. J Ind Microbiol Biotechnol 2006, 33, 859-868.

(4) Yu, F.; Zhu, X.; Du, L. Fems Microbiol Lett 2005, 248, 257-264.

(5) Bojja, R. S.; Cerny, R. L.; Proctor, R. H.; Du, L. J Agric Food Chem 2004, 52, 2855-2860.

(6) Kongsaeree, P.; Prabpai, S.; Sriubolmas, N.; Vongvein, C.; Wiyakrutta, S. J Nat Prod 2003, $66,709-711$. 